\title{
Mite Infestations of Man Contracted from Dogs and Cats
}

\author{
L. R. THOMSETT,* M.R.c.v.s.
}

\begin{abstract}
Summary.-Acarine infestations of the dog and cat $N$ are transmissible to man. The relation between age incidence in the host, duration of disease, and circumstances under which the animal is kept are stated. Fifty out of 65 human contacts at risk to 42 infected dogs and cats showed lesions of mite infestation ; $48 \%$ of these lesions were confined to the arms and torso.

It is important to consider animal mite infestation in the differential diagnosis of human pruritic and papular skin disease.
\end{abstract}

\section{Introduction}

Mite infestations of the skin of small domestic animals which are transmissible to man are common in the United Kingdom. Human contact with infested animals readily gives rise to skin lesions for which medical advice may be sought. The purpose of this communication is to indicate the parasites involved in animal disease which may give rise to human infestation. With the marked increase in the incidence of human scabies it is of importance to include animal mite infestations in the differential diagnosis of human pruritic skin disease.

\section{Acariasis in Animals}

Sarcoptes scabiei var. canis commonly infests dogs, while Cheyletiella parasitovorax, a free-living mite in the hair coat of the dog, cat, and rabbit, does so to a lesser extent. Both parasites are, however, readily able to cause skin disease in man.

Notoedres cati, the counterpart in the cat of S. scabiei in the dog, has been recorded as the cause of a papular skin condition in man (Davies, 1941). In Britain feline acariasis due to this mite is now almost unheard of. Occasionally the condition may be encountered in cats living a semi-wild existence, and such animals rarely permit handling by man. Human infestation with $N$. cati is therefore unlikely to occur.

Direct infestation of man with the larval forms of the harvest mite Trombicula autumnalis is well recognized. The dog and cat are seasonally infected with this acarus and may act as mechanical vectors of it to man, resulting in human pruritic skin disease.

Sarcoptic acariasis.-This disease in the dog begins as a highly irritant papular rash affecting the thin-skinned areas of the body. The lesions, as in man, are produced by ovigerous female acari burrowing into the stratum corneum in order to lay their eggs. Self-inflicted injury by scratching, gnawing, and rubbing the sites of infestation leads to chronic thickening of the skin, exudation of serum and blood, with subsequent crust formation. Secondary bacterial infection leads to the formation of pustules.

In the absence of early diagnosis and treatment, extension of the lesions to involve the whole of the animal occurs with the development of a patchy alopecia accompanying the lesions described.

Department of Medicine, Royal Veterinary College, North Mimms, Hatfield, Herts.
In dogs with a dense hair coat early lesions may be imperceptible on superficial examination, and, apart from pruritus, the animal is symptom-free. Not until areas of hair loss and obvious lesions become apparent do owners seek advice, often many weeks after the beginning of the disease process.

Chronic scabies associated with a low-grade infestation is common after inadequate treatment, and in untreated animals which become tolerant to the infestation it may persist for many years.

Lesions of chronic infestation are commonly confined to the fringe of the pinna of the ears and the skin of the lateral aspect of the elbows and hocks-seen as chronic skin thickening, minimal crust formation, and persistent mild itch.

As in man the pruritus associated with scabies in the dog is increased with a rise in environmental temperature and is particularly noticeable in animals kept in centrally heated homes or when they are lying in front of a fire.

Sarcoptic scabies in the dog has usually been associated with young animals, in particular puppies (Smith and Claypoole, 1967), though the average age of patients in this survey was 44.7 months, $71 \%$ being within the 1 to 48 months' age group.

\section{Acariasis Due to C. parasitovorax}

Infestation of the hair coat of the dog or cat with C. parasitovorax may or may not cause skin disease in the host, though the animal remains a potential source of infection to human contacts. C. parasitovorax is a free-living mite (Fig. 1) which may be distinguished from other members of the Cheyletidae by terminal combs on the legs. The adult mite is just visible to the naked eye. The life-cycle of this species is spent entirely within the hair coat of the host. The eggs, which are attached to the hair shafts by means of a

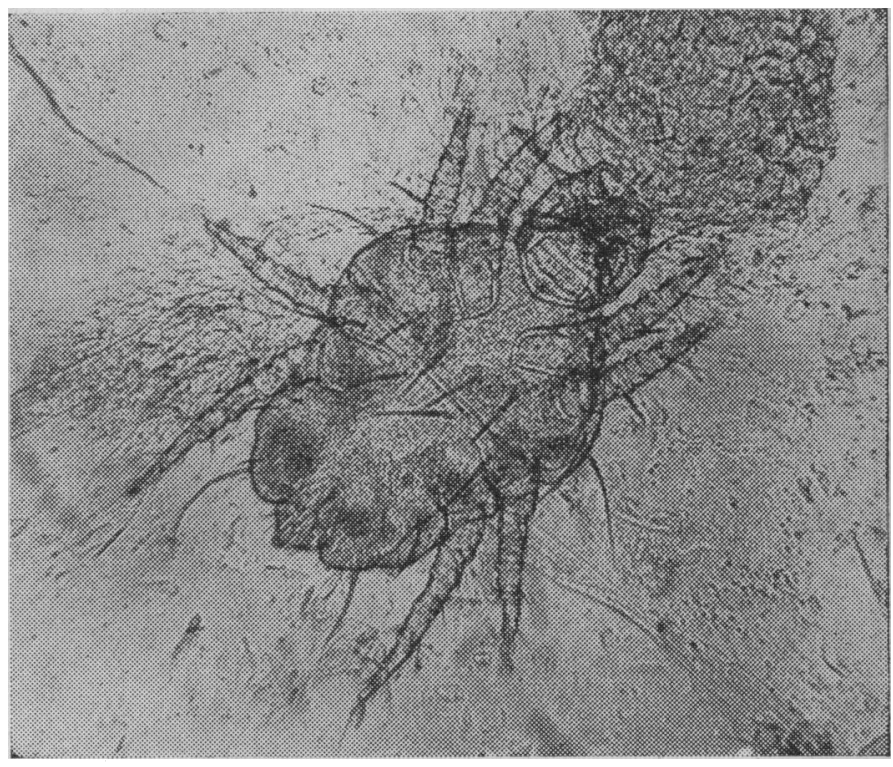

FIG. 1. - C. parasitovorax. $\times 64$ 
short thread-like process, hatch in four days, and after three moults the life-cycle is completed in 34 days (Humphreys, 1958).

Lesions on the animal host vary considerably, depending on the density of infestation and response of the host. In the dog the predilection site appears to be the skin of the dorsum between the base of the neck and root of the tail, though generalized infestations occur. In the cat and rabbit the distribution is more generalized throughout the hair coat.

In the dog a mild infestation with $C$. parasitovorax leads to pruritus and hyperaesthesia of the skin of the dorsum with no other evidence of disease. Heavy infestation causes skin hyperaesthesia, persistent pruritus with scratching and selfinflicted injury, a papular rash, and excessive production of dandruff (Hart and Malone, 1958). Secondary bacterial infection is not a feature.

The duration of disease in the animal cases under review and the age incidence are indicated in Fig. 2.

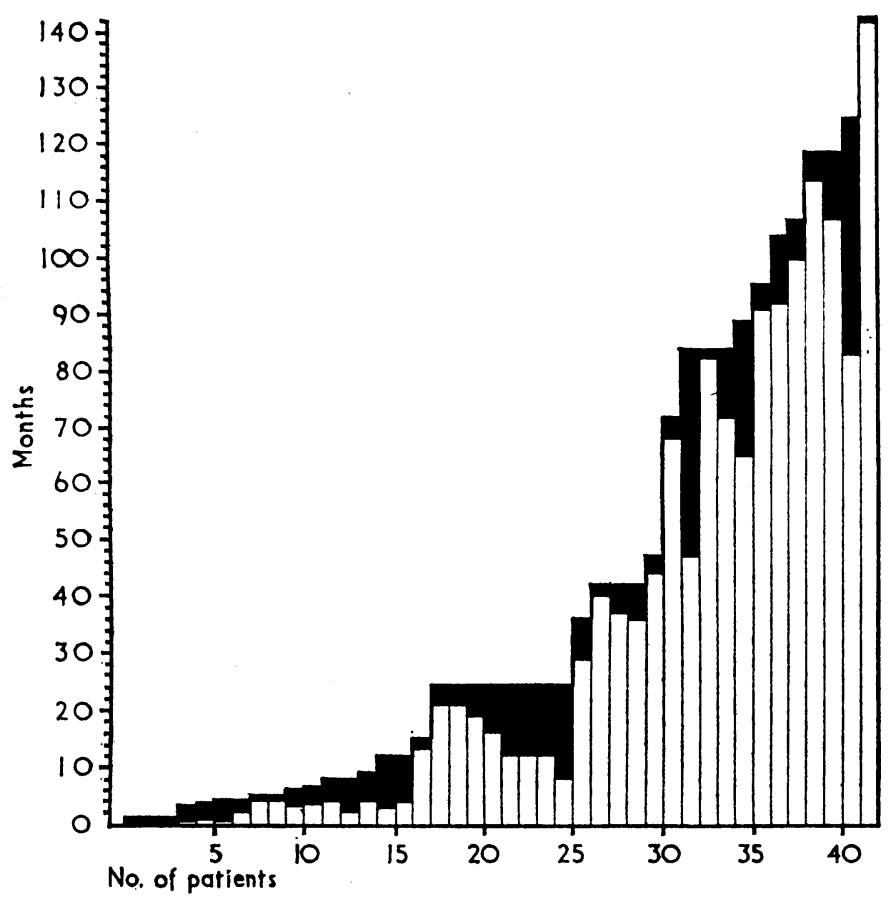

Fig. 2.-Age incidence and duration of mite infestation in animals. The hatched area indicates the time at which infestation occurred and duration up to the time of investigation, in months.

\section{Diagnosis}

The diagnosis of animal infestations with S. scabiei var. canis and $C$. parasitovorax depends on the demonstration of the parasite. Direct microscopical examination of skin scrapings cleared in liquor potassae is often unsatisfactory in the diagnosis of scabies, concentration and sugar flotation procedures being more reliable.

Since $C$. parasitovorax is a free-living mite the examination of coat brushings of dandruff and coat debris is often adequate for diagnosis. In heavy infestations mites may be seen with a hand magnifier, but where infestation is minimal diagnosis may require the use of concentration methods as used in the diagnosis of scabies.

\section{Human Infestation with Dog and Cat Mite}

In the course of consultant dermatological practice at the Royal Veterinary College many cases of chronic skin disease of the dog and cat are investigated, a proportion of which are referred because of suspected human involvement. The majority of cases where human skin disease has been related to an infected animal have been investigated at the request of the owner through his attending veterinarian and not at the instigation of the owner's medical adviser.

In the investigation of animal dermatological problems it is my routine practice to determine whether owners of affected animals are suffering from concurrent skin disease and whether medical advice has been sought. In the majority of cases where human involvement is confirmed no medical advice has apparently been sought, and it is remarkable that an owner with animal scabies or a cheyletiella mite rash will endure this for a considerable time, in some cases years, before seeking a professional opinion for themselves or their animal.

In the present survey 65 human contacts were directly at risk to 42 dogs or cats kept as household pets or as part of a commercial breeding enterprise. Twenty-eight dogs were infected with S. scabiei var. canis; 12 dogs and two cats were infected with $C$. parasitovorax. All diagnoses of infected animals were confirmed by demonstration of the causal parasite. Of the 65 human contacts 50 showed lesions of mite infestation, 16 being due to $C$. parasitovorax and 34 to S. scabiei var. canis.

The incidence of human infection was related to the circumstances under which the infested animal was kept and to the animal's size. Thirty dogs of small breeds, all of which were kept within the domestic household and readily handled by both adults and children, were incriminated in the transmission of infestation from animal to man. Only one of four persons breeding dogs commercially complained of personal infestation or of infection of their staff with animal mites.

Lesions in man vary from an irritant papular rash to a severe sensitivity response with blister formation (Davies, 1941; Lomholt, 1947 ; Fernström and Gentele, 1960) on the arms, torso, neck, and legs. The sites of predilection are the arms and torso, in particular the waistline, usually associated with nursing affected animals on the lap. It should be noted that human infestation may occur either by direct contact with an infected animal or by migration of the parasites from the animal through clothing; immediate contact between the skin of the animal and man is not therefore a prerequisite for human infestation. The site distribution for lesions in 50 persons was :

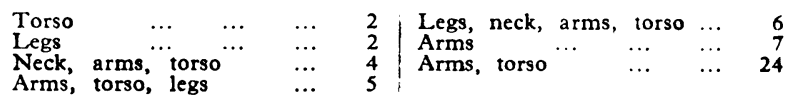

Confirmation of diagnosis in man by demonstration of the causal parasite presents considerable difficulty inasmuch as S. scabiei var. canis, though producing lesions, is said not to establish itself and burrow in human skin. A similar difficulty arises with $C$. parasitovorax owing to its free-living life-cycle. Since it is only with the greatest difficulty that these parasites may be found on human skin, confirmation of animal acariasis in man depends entirely on the demonstration of the organism on the animal host.

Two examples of case histories in animal owners are given below.

\section{Case 1}

A woman purchased a small pet dog 4 months of age from a reputable supplier. The animal had the freedom of the house but had its own sleeping quarters. A contact Siamese cat was also kept. When the dog was $2 \frac{1}{2}$ years of age the owner complained of an intermittent papular rash on her body, arms, and legs, considered on medical opinion to be emotional in origin and treated 
accordingly. The condition recurred, and at each recurrence the lesions were more severe. Veterinary examination of the dog showed no lesions of skin disease though there was a tendency for the animal to scratch itself more often than normal.

This situation continued for a further 18 months, during which time the owner underwent psychiatric investigation and treatment for her skin disease, and on medical advice took a holiday with her family, accompanied by the dog. On the return journey by car, having nursed the dog, her skin disease became severe, with lesions consisting of large blisters, widely distributed about the anterior torso, arms, and thighs. Having so definitely nursed the dog she then considered the possibility of disease acquired from the animal.

Examination of the animal by her veterinarian showed little other than hyperaesthesia of the dorsal skin and small accumulations of bran-scale dandruff and a small area of dorsal alopecia. The dog was referred to me for further investigation. Examination of the animal showed it to be a well-nourished 4-year-old male pug dog. Apart from an area of hair loss approximately $2 \mathrm{~cm}$. in diameter over the dorsal midline between the scapulae, bran-scale dandruff, and a rather more than normal tendency to moult, little of significance was noted. Manipulation of the hair tips over the dorsum and flanks showed significant skin hyperaesthesia with reflex scratching, a feature of canine infestations with $C$. parasitovorax. Coat brushings and skin scrapings taken from the affected area of the dorsum showed large numbers of $C$. parasitovorax in various stages of its life-cycle. Eggs of this parasite were demonstrated on epilated hairs. Repeated treatment of the dog with Tetmosol (monosulfiram) soap was prescribed, and the infestation cleared within three weeks.

The owner, of her own volition, bathed herself with the same preparation, and instructed all other members of her household to do likewise. She sent her clothing for dry-cleaning, together with such house furnishings with which the dog had been in contact and were removable. The dog's bed was destroyed. Within one week she was symptom-free and has subsequently remained so.

\section{Case 2}

A woman purchased a puppy which was subsequently given the freedom of the house. The dog showed evidence of a papular rash on the abdomen which spread to the limbs; lesions later became crusted and a patchy alopecia with persistent pruritus became an established feature of the condition. The woman's son was permitted to have the dog in bed with him, and was found by the parent to be affected with an irritant papular rash of the body, arms, and !egs, particularly the upper chest. The woman was similarly affected on the forearms and around the waist.

Treatment for the dog's skin cisease had been carried out on the advice of the owner's veterinarian, but owing to the suspected human involvement the animal was referred to the Royal Veterinary College for a further opinion and investigation.

Examination of the 5-month-old male puppy showed papular and crusted lesions affecting the whole of the body and limbs. There was a patchy alopecia with excess moulting of hair. Pruritus was a significant symptom, and areas of lichenified skin with accumulated dandruff were evident on the flanks and hindquarters. Skin scrapings confirmed the condition as sarcoptic scabies.

Treatment of the animal with benzene hexachloride shampoo was instituted, and the owners were advised to avoid contact with the dog as much as possible until the condition was cured.
Spontaneous remission of the human skin disease was subsequently reported.

\section{Differential Diagnosis of Acariasis in Animals and Man}

Human infection with the skin parasites of small domestic animals, though recognized, is rarely considered of importance. Smith and Claypoole (1967) suggested that there is a need for closer consideration of such forms of parasitism in the differential diagnosis of papular and vesicular skin disease in man; that this is so is borne out by the experience reported here. With a population of at least 10 million dogs and cats, approximately $50 \%$ of each species, kept in households in the United Kingdom and an incidence of parasitism with S. scabiei alone in the dog of approximately $1 \%$-that is, 50,000-one would anticipate the number of human cases to be significant.

The problem of the differential diagnosis of scabies in the $\operatorname{dog}$ is less difficult than in man. Since in the majority of cases in the dog the acarus has a predilection for the fringe of the pinna of the ears, a scratch reflex can be elicited on manipulation of this site which is pathognomonic of scabies. In the few cases where the ear is not involved, and in cheyletiella infestations of both dog and cat, differentiation between parasitism due to other organisms and other papular rashes or disorders which give rise to excess dandruff present difficulty. The confirmation of mite infestation therefore depends finally on the demonstration of the causal parasite. Skin disease in the dog and cat of emotional origin is of little consequence, though scratching and self-mutilation may be associated with boredom.

In man differential diagnosis of skin disease contracted from animal contact presents greater problems, for neither $S$. scabiei var. canis nor $C$. parasitovorax is readily demonstrable in man. Lyell (1967) discusses specific differentiation of pruritic skin disease, and states that emotional factors among others are readily invoked as the cause of pruritus in man. For the purpose of differential diagnosis it should be borne in mind that, though this communication deals only with mite infestations of the dog and cat, other domestic species-for example, cage birds, poultry, and horses-may also be vectors of arthropods able to cause papular skin disease in man.

\section{Treatment}

It is obvious that treatment of the offending animal or its banishment from the household is much to be preferred to treatment of the owner with tranquillizers, sedatives, antihistamines, or even the consultant psychiatrist's couch.

\section{REFERENCES}

Davies, J. H. T. (1941). Brit. 7. Derm., 53, 18.
Fernström, A. J. B., and Gentele, H. (1960). Acta paediat. (UPpsala), 49, 752.

Hart, C. B., and Malone, J. C. (1958). Vet. Rec., 70, 991.

Humphreys, M. (1958). Vet. Rec., 70, 442 .

Lomholt, S. (1947). Ugeskr. Lag., 109, 228.

Lyell, A. (1967). Brit. med. f., 2, 223.

Smith, E. B., and Claypoole, T. F. (1967). F. Amer. med. Ass., 199, 59. 